\title{
Transient quadriplegia after fluoroscopic-guided selective cervical nerve root block in a patient who received cervical interbody fusion
} -A case report-

\author{
Mi Hyeon Lee, Young Deog Cha, Jang Ho Song, Young Mi An, Jeong Uk Han, and Du Ik Lee \\ Department of Anesthesiology and Pain Medicine, Inha University Hospital, Incheon, Korea
}

Selective cervical nerve root block is executed for patients who have symptoms of cervical radiculopathy for diagnostic and therapeutic purposes. However several catastrophic complications caused by this procedure have been reported including neurological complications. A 43-year-old male received a C5 selective cervical nerve root block procedure due to continuous radiating pain even after cervical discectomy and interbody fusion was performed. At the time of the procedure, the contrast outline revealed reflux of the nerve root and epidural space. But after the procedure was performed, the patient experienced decreased sensation in the upper and low extremities as well as motor paralysis of both extremities. Our sspecting diagnosis was anterior spinal artery syndrome but both sensory and motor functions were subsequently recovered within a few hours after the procedure was completed. Due to the difficult nature of this case, we reported these complications and reviewed current literature related to this study. (Korean J Anesthesiol 2010; 59: S95-S98)

Key Words: Anterior spinal artery syndrome, Intrathecal injection, Paralysis, Spinal nerve root.

Selective cervical nerve root block is chosen for diagnosis or treatment for cervical radiculopathy. It can be executed even in a case where cervical radiculopathy is not resolved after surgery such as in cervical discectomy or interbody fusion procedures. According to a report encompassing 1,036 cases of performing continuous fluoroscopic-guided extraforaminal cervical nerve blocks [1] and a prospective study by Pobiel et al. [2], selective cervical nerve root block was described as a relatively safe operation. However, there have been reported critical neurological complications related to this operation such as anterior spinal artery syndrome or cerebral infarction [3]. In addition, the possibility of post-operative anatomical changes will be expected to increase such risk. We observed an episode of the incidence and recovery of hypoesthesia and motor

Received: May 11, 2010. Revised: 1st, May 11, 2010; 2nd, May 27, 2010. Accepted: July 7, 2010.

Corresponding author: Young Deog Cha, M.D., Department of Anesthesiology and Pain Medicine, Inha University Hospital, 7-206, Shinheungdong 3ga, Jung-gu, Incheon 400-711, Korea. Tel: 82-32-890-3968, Fax: 82-32-881-2476, E-mail: ydchaan@inha.ac.kr This work was supported by Inha University.

(c) This is an open-access article distributed under the terms of the Creative Commons Attribution Non-Commercial License (http:// creativecommons.org/licenses/by-nc/3.0/), which permits unrestricted non-commercial use, distribution, and reproduction in any medium, provided the original work is properly cited. 
paralysis of limb in a patient who underwent a C5 selective cervical nerve root block to treat his persistent post-operative cervical radiculopathy.

\section{Case Report}

A 43-year-old male patient with cerebral palsy (athetoid type) was diagnosed with cervical disc herniation in addition to a symptom of 1-year history of pain radiating to the upper limb. The patient underwent a laminectomy (C3-5), an anterior interbody fusion (C3-5), a posterior interbody fusion (C3-5), and an artificial bone graft operation 1 year ago. However, he complained of persistent pain mediated by the branch of the left C5 after the operations. He was then referred to the Pain Clinic of our hospital for inpatient collaborative consultation for treatment. At that time, his visual analogue scale (VAS) score was an 8 out of 10. After due consideration that it was difficult to perform interlaminar epidural block because of the inability to put the patient in the appropriate position and that the stellate ganglion block had no effect, a left C5 selective cervical nerve root block was performed.

Monitoring devices were set to measure the patient's electrocardiogram (EKG), blood pressure (BP), and arterial oxygen saturation $\left(\mathrm{SpO}_{2}\right)$. He was placed into the supine position and the puncture area was disinfected with the broad application of betadine solution on the neck and was covered with a sterile gauze dressing. Under continuous radiography, while having the neck held to the anterior-superior and adjusting c-arm fluoroscopy to 45 degrees, a needle was advanced via the anterolateral approach. When a $5 \mathrm{~cm}$-block needle

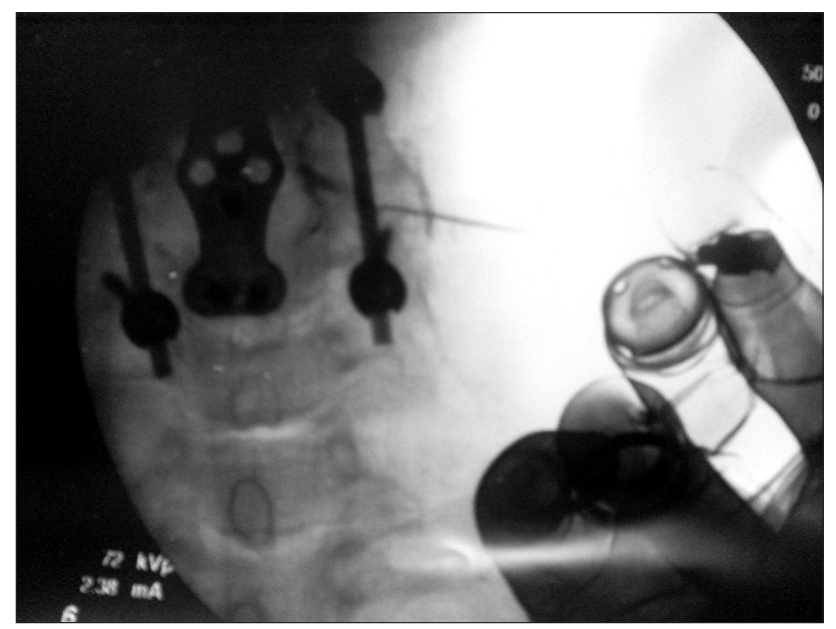

Fig. 1. An anterior-posterior view of cervical spine during C5 nerve root injection. The needle lies in the C5-6 intervertebral foramen after injection of $1 \mathrm{ml}$ contrast medium. The needle is not advanced no further than halfway across facet column. The contrast outline shows the nerve root and epidural reflux taking place. reached the superior articular process of the left C5 and was advanced to the neural groove, care was taken to confirm that placement of the needle tip did not across the halfway point of the posterior cervical articulate pillar. After injection of 1.5 $\mathrm{ml}$ contrast medium, radicular and epidural contrasts were confirmed, but vascular or intraspinal contrasts were not observed (Fig. 1). When the patient complained of paresthesia caused by injection needles, a mixed solution of $3 \mathrm{ml} 1 \%$ mepivacaine and $40 \mathrm{mg}$ triamcinolone was infused. Prior to the infusion, no body fluids such as regurgitated blood or cerebrospinal fluid were observed with aspiration.

Approximately 2 minutes after administration, the patient felt weak in the right arm as well as the left arm, however when his motor ability was grade 3 of 5 in both upper limbs, all senses regarding pain, temperature, and position disappeared. One minute later, the motor ability of both upper limbs was entirely gone, immediately followed by disappearance of the sensation and motor ability of the lower limbs, and the patient complained of labored respiration. At that time, his BP was $150 / 90 \mathrm{mmHg}, \mathrm{SpO}_{2}$ at pulse oximetry was $98 \%$, and EKG indicated a normal sinus rhythm. As his anxiety and breathing difficulty worsened, midazolam $2 \mathrm{mg}$ was administered and the patient's respiration was assisted with $100 \%$ oxygen. His spontaneous breathing was maintained, so that positive pressure ventilation was not employed. Within 45 minutes he awakened, with no signs of labored respiration, and when his motor ability was was measured, it showed grade 3 at the upper limbs, grade 2 at the lower limbs, which revealed signs of mild recovery. His temperature, pain, position, and vibration senses were all fully recovered.

2 hours after the patient was transferred to the postanesthetic care unit (PACU), he regained normal motor ability and sensation completely. When his vital signs were confirmed as normal, he was transferred to the general ward.

\section{Discussion}

Continuous fluoroscopic-guided cervical nerve root block is used as an non-invasive alternative to surgical treatments and is also used for pre-operative diagnosis to determine dororific branch. There have been various opinions with regards to the safety of selective cervical nerve root block. Reports by Ma et al. [1] and Pobiel et al. [2] describe this operation as relatively safe. On the other hand, a study without specific complications recognized potential complications such as penetration to the vital vessels or dural puncture [4], while there has been an additional report on neurological complications including cerebral and spinal cord infarctions [5].

On selective cervical nerve root block following spine surgery as in the present case, neurological sequela is hard to remove 
completely even though nerve block is performed with care, and medication is inadvertently injected into the wrong place although radicular and epidural contrasts were successfully achieved.

As for the development of quadriplegia during cervical nerve root block, we suspect the following causes: steroid particleinduced anterior spinal artery syndrome, and subdural or intraspinal injection of medicines.

Anterior spinal artery syndrome is usually seen as a result of infarction along the front of the spinal cord when steroid particles are injected to the vertebral artery or the radicular artery. Although BP does not alter and vibration sensation and motion function are retained, the pain and temperature senses are suppressed with complete motor paralysis. Even after all sensations are recovered, the motor function does not return to normal [3]. The continuous fluoroscopic images of the present case did not show any angiogram suspicious of intravascular injection or blood aspiration of the needles. However, Barker et al. [6] claimed that infusion of drugs into the radicular artery even in a continuous fluoroscopic-guided operation performed with care may cause anterior spinal artery syndrome. In a study using 0.5-2 ml contrast medium, Furman et al. [7] reported that the overall rate of intravascular contrast injections was $19.4 \%$ and that observing blood in the needle hub could predict intravascular injection with a specificity of $97 \%$, but with sensitivity of only $45.9 \%$. In addition, Hwang et al. [8] reported the overall intravascular injection rate of $63.4 \%$ in a study using $3 \mathrm{ml}$ of contrast medium. Therefore, when patients complained of labored respiration, decreased sensation of temperature and pain, and decreased motor function, the possibility of anterior spinal cord infarction caused by the intravascular infusion of steroid particles was initially suspected. In the present case, however, the patient regained normal breathing within 2 hours and recovered motor function as well as sensation. Therefore, we excluded the possibility of anterior spinal cord infarction.

Another factor to cause quadriplegia would be subdural injection. Contrast patterns of subdural injection are manifested as an opaque bulging in the front of the vertebral canal and symptoms of a subdural injection (block) include a delayed onset varying from 5 to 30 or more minutes [9]. In the present case, however, contrast images did not hint at subdural injection, while quadriplesia showed up immediately within 2 minutes, unlike the case of subdural injection.

Complete or partial subarachnoid injection can be hypothesized to cause quadriplegia. For the patient's clinical presentations, the onset of the symptom occurred within 2 minutes and his sensation and motor function were recovered in 45 minutes, which led us to assume intrathecal injection of medication was the primary cause of transient quadriplegia. During the procedure, however, radiographic findings showed clear images of radicular and epidural contrasts, the placement of the needle did not pass over middle of the facet column, and cerebrospinal fluid (CSF) was not detected at aspiration, either. The patient's vital signs also did not show decrease of BP nor bradyrhythmia. Therefore, at the initial stage of the symptom, we did not suspect intrathecal injection of medication. On the contrary to our case, Brouwers et al. [3] mistook the initial symptom of anterior spinal artery syndrome as intrathecal injection of medication. The reasons that subarachnoid injection took place without any abnormal findings on contrast images in the present case may be as follows: first, inserted instruments may have obscured the intrathecal contrasts produced by the injected contrast medium for pilot guide; second, possible anatomical changes such as adhesion resulting from the operation itself may have caused the needle to be placed subdurally; third, even though the needle was not placed inside the dura, spread of medication through ripped regions of the dural sleeves induced by the operation might have caused subarachnoid injection. Anatomically, the dural sleeve is tethered to the transverse process and dura is surrounded from the nerve root's take-off point out of the spinal cord to the foraminal lateral boundary [10]. After operation, anatomical alteration may occur due to adhesion by the surrounding tissues. Kuslich et al. [11] reported that the incidence rate of dural tears after spinal interbody fusion was 3 to $10 \%$. In a study of Chen et al. [12] involving 118 patients, the incidence of dural tears was $3.4 \%$, among which $3 / 4$ of the incidences were found in the axilla of the nerve root. This region has been known for its difficulty of restoration after it is damaged. In the current case, intrathecal injection of contrast medium was not observed. Therefore, intrathecal spread of contrast medium through ripped regions of the axilla of the nerve root induced by operation seems to be a more plausible mechanism of transient quadriplegia rather than direct intrathecal injection of the medication. Because computed tomography (CT) could not confirm the soft tissue, however, an exact explanation is difficult to compose based on the process of intrathecal injection of medication.

A recent publication suggests a guideline of securing the safety of the cervical nerve root blocks: first, facilitate real-time c-arm fluoroscopy; second, administer a test dose of contrast medium; third, place the needle toward the posterolateral side of the foramen to avoid inadvertent intravascular injection; fourth, at performing procedure, use the anterolateral approach to the cervical spine [3]. According to anatomical studies, the radicular artery runs to the front of the vertebral nerves, but the feeding arteries are present anywhere. Therefore, it needs realtime monitoring of contrast medium and confirmation of the soft tissue [13]. Computed tomography (CT) and CT fluoroscopy are useful in confirming the presence of the soft tissue, but 
they are not reliable for consistent monitoring. Therefore, using digital subtraction should be considered [6]. In light of the study by Hwang et al. [8], it is further recommended that use of more than $3 \mathrm{ml}$ of contrast medium could be helpful in identifying wrong placement of the needles (e.g., unintended intravascular injection).

To enhance the safety of the procedures, choice of medications should also be taken into account. In the present case, the patient had long-standing post-operative pain, and stellate ganglion block or interlaminal cervical epidural block was difficult to perform. Although cervical nerve root block was reluctantly decided upon, the procedure was not easy to perform due to post-operative anatomical alteration. Additionally, medication was needed that could extend topical effects. As an option, soluble steroid is well known for its capacity of rapid intraspinal clearing effect with short duration. Another option would be triamcinolone, which is most frequently used as a topical agent for its anti-inflammatory effects, less sodium retention, and long-duration potency [14]. Therefore, we chose triamcinolone. Because particulate steroids can induce arachnoiditis when intrathecally injected and they can cause critical complications such as spinal cord infarction or cerebral infarction when intravascularly injected, Baker et al. [6] suggested utilization of a test injection of non-steroidal local anesthetic. Numerous reports have recommended particlefree steroids as a potential remedy. In particular, Lee et al. [15] argued that there were no differences between particulate steroids and non-particulate steroids in a study of comparing dexamethasone and triamcinolone. Therefore, it should be considerable to use dexamethasone as a non-particulate steroid in order to reduce the risks of performing the procedures.

In conclusion, anesthesiologists should keep in mind that complications unidentifiable on contrast images alone are potentially present at cervical nerve root block immediately following spine surgery. Therefore, when a procedure for a post-operative patient is necessary, it is critical to administer a test dose of local anesthetic, to use enough amount of contrast medium, to facilitate real-time monitoring devices, and to employ fluoroscopy with digital subtraction to check the soft tissues, while exerting a careful observation of the patient's symptoms at administration of medication.

\section{References}

1. Ma DJ, Gilula LA, Riew KD. Complications of fluoroscopically guided extraforaminal cervical nerve blocks: an analysis of 1036 injections. J Bone Joint Surg Am 2005; 87: 1025-30

2. Pobiel RS, Schellhas KP, Eklund JA, Golden MJ, Johnson BA, Chopra $S$, et al. Selective cervical nerve root blockade: prospective study of immediate and longer term complications. AJNR Am J Neuroradiol 2009; 30: 507-11.

3. Brouwers PJ, Kottink EJ, Simon MA, Prevo RL. A cervical anterior spinal artery syndrome after diagnostic blockade of the right C6nerve root. Pain 2001; 91: 397-9.

4. Kikuchi S, Macnab I, Moreau P. Localization of the level of symptomatic cervical disc degeneration. J Bone Joint Surg Br 1981; 63: 272-7.

5. Ludwig MA, Burns SP. Spinal cord infarction following cervical transforaminal epidural injection: a case report. Spine 2005; 30: E266-8.

6. Baker R, Dreyfuss P, Mercer S, Boqduk N. Cervical transforaminal injection of corticosteroids in8to a radicular artery: a possible mechanism for spinal cord injury. Pain 2003; 103: 211-5.

7. Furman MB, Giovanniello MT, O'Brien EM. Incidence of intravascular penetration in transforaminal cervical epidural steroid injections. Spine (Phila Pa 1976) 2003; 28: 21-5.

8. Hwang SJ, Han KR, Kim SY, Kim NS, Kim C. Analysis of intravascular flow patterns following cervical transforaminal epidural injection. Korean J Pain 2009; 22: 52-7.

9. Goodman BS, Bayazitoglu M, Mallempati S, Noble BR, Geffen JF. Dural puncture and subdural injection: a complication of lumbar transforaminal epidural injections. Pain Physician 2007; 10: 697-705.

10. Pierre B. The cervical spine. In: A system of orthopaedic medicine. 2nd ed. Edited by Ludwig O, Pierre B, Herman J: Singapore, Churchill \& Livingstone. 2003, pp 135-46.

11. Kuslich SD, Ulstrom CL, Griffith SL, Ahern JW, Dowdle JD. The Bagby and Kuslich method of lumbar interbody fusion. History, techniques, and 2-year follow-up results of a United States prospective, multicenter trial. Spine (Phila Pa 1976) 1998; 23: 1267 78.

12. Chen L, Tang T, Yang H. Complications associated with posterior lumbar interbody fusion using Bagby and Kuslich method for treatment of spondylolisthesis. Chin Med J (Engl) 2003; 116: 99-103.

13. Wagner AL. CT fluoroscopic-guided cervical nerve root blocks. AJNR Am J Neuroradiol 2005; 26: 43-4.

14. Abram SE. Treatment of lumbosacral radiculopathy with epidural steroids. Anesthesiology 1999; 91: 1937-41.

15. Lee JW, Park KW, Chung SK, Yeom JS, Kim KJ, Kim HJ, et al. Cervical transforaminal epidural steroid injection for the management of cervical radiculopathy: a comparative study of particulate versus non-particulate steroids. Skeletal Radiol 2009; 38: 1077-82. 